Article

\title{
Evolutionary Coalitional Game Based Cooperative Localization in Vehicular Network
}

\author{
Ting Yin ${ }^{1,2 *}$, Decai Zou ${ }^{1,2}$, Xiaochun $\mathrm{Lu}^{1,2}$, and Cheng Bi ${ }^{3}$ \\ 1 National Time Service Center, Chinese Academy of Sciences, Xian 710600, China; yinting@ntsc.ac.cn (T. \\ Yin); master006@163.com(D. Zou); 1xc@ntec.ac.cn(X. Lu); \\ 2 University Chinese Academy of Sciences, Beijing 100049, China; \\ 3 Xidian University, Xian 710000, China; bicheng_pro@126.com \\ * Correspondence: yinting@ntsc.ac.cn
}

\begin{abstract}
Cooperative localization under complex urban environments has become a solution to replace the Global Navigation Satellite System (GNSS) positioning. Due to utilizing distance measured and information exchanged between vehicles, cooperative localization results in high computational complexity and heavy communication overhead. This paper proposes a cooperative localization method based on evolutionary coalitional game theory, which implements vehicles' location estimation with less communication cost. We select the neighboring vehicles to form a coalition based on the node's square position error bound and communication cost. The location is obtained via exchanging information between vehicles. It is evident from the simulations and results that the proposed method holds low communication overhead while maintaining localization accuracy.
\end{abstract}

Keywords: Cooperative localization; Vehicle network; Game theory; Evolutionary coalitional game.

\section{Introduction}

As the Intelligent Transportation System (ITS) develops rapidly, vehicle positioning technology has become the focus of attention for autonomous driving applications [1-2]. The vehicle is generally located with global navigation satellite systems (GNSS). However, the signals of GNSS are usually poor in urban canyons and overpasses due to highrise building occlusion and multipath. Therefore, the demand for localization accuracy under dense and complex urban environments has become imminent.

There are many novel localization methods in vehicle networks to deal with complex urban environments. Visual localization is a common technology to match and predict vehicles' position via identifying and searching local features [3]. Similarly, radar technology is widely used in dual-functional radar-communication (DFRC) systems, in which it is superior to reducing the signaling overhead and improving the accuracy of the angle estimation [4]. Since different positioning technologies are suitable for urban environments, multi-data fusion has become an inevitable trend. The fusion of multiple localization information can effectively deal with the complex urban environment [5-6].

With the development of the fifth generation of mobile communication (5G), 5G mmWave positioning has become a new localization scheme [7], which is generally categorized in the base station (BS) based and user-based [8]. The BS-based method requires Location Management Function to estimate users' position. However, the vehicles fail to locate due to network congestion and high latency in dense urban environments and crowded situations when many vehicles access the BS. Inversely, the user-based is more suitable than BS for vehicle networks. Besides, cooperative vehicle localization plays an effective model for the user-based [9-11]. In general, non-Bayesian-based and Bayesian-based are considered for cooperative localization. Widespread estimators are 
contained the least-squares (LS) based and the maximum likelihood (ML) in the nonBayesian-based model.

Similarly, message propagation and non-message propagation are present for Bayesian-based [12-13]. It is noticed that the Bayesian-based performed well with the non-Bayesian method in terms of localization accuracy. The classical algorithm of the Bayesian-based method is the Sum-product algorithm over a wireless network (SPAWN) [12], which estimates the position via the anchors' positioning information and the posterior probability of the nodes through message passing. In [14], the authors employ the idea of the posterior linearization filter in belief propagation with the sigmapoint method. The posterior linearization belief propagation (PLBP) performs the localization accuracy well with the nonlinear measurement. However, the cost of communication and computational complexity increase under the scenarios with numerous nodes, and it cannot meet the requirement of use cases.

The relevant problems have lately received considerable attention in cooperative localization. The role of game theory is successful in solving the limitations. In [15], the authors propose an improved game theory-based co-localization algorithm for the multirobot system localization. In [16], the authors propose a network formation game for cooperative localization to reduce computational complexity and resultant network traffic. Similarly, the coalition game, a typical static game theory, is widely implemented for cooperative localization [17]. In addition to that, the most informative agents to exchange information is determined with opportunistic cooperative localization-based overlapping coalition game [18-19]. However, since the static game needs replay to reach equilibrium with heavy communication overhead every time the topology changes, it is unsuitable for dynamic network topology like vehicle networks. Therefore, we embed the dynamic game theory in cooperative localization and propose a framework to solve the problem of communication cost.

The main contribution of this paper is to address the problem of high computation complexity and communication cost for vehicle networks in cooperative localization. In this paper, we formulate the cooperative vehicle localization problem as an evolutionary coalitional (EC) game. The vehicles autonomously decide whether to assist other vehicles based on their positioning performance and the current position relationship. The simulation results demonstrate that the proposed method performs better than the PLBP algorithm. It is noticed that the proposed method reduces the computation complexity and communication cost while maintaining the same accuracy.

\section{Materials and Methods}

\subsection{Network Model}

The crossroads congestion scenarios in the city are described in Fig.1. Due to dense high buildings, the vehicles cannot receive satellite signals with good Geometric Dilution Precision. The scenario is composed of M Roadside unit (RSUs) and N vehicles. In detail, the RSU is considered a small base station with precise location and clock information, and the vehicles are within the coverage of the roadside unit. Specially, the vehicle to be time-synchronized with the RSU, and randomly deployed with unknown positions. 


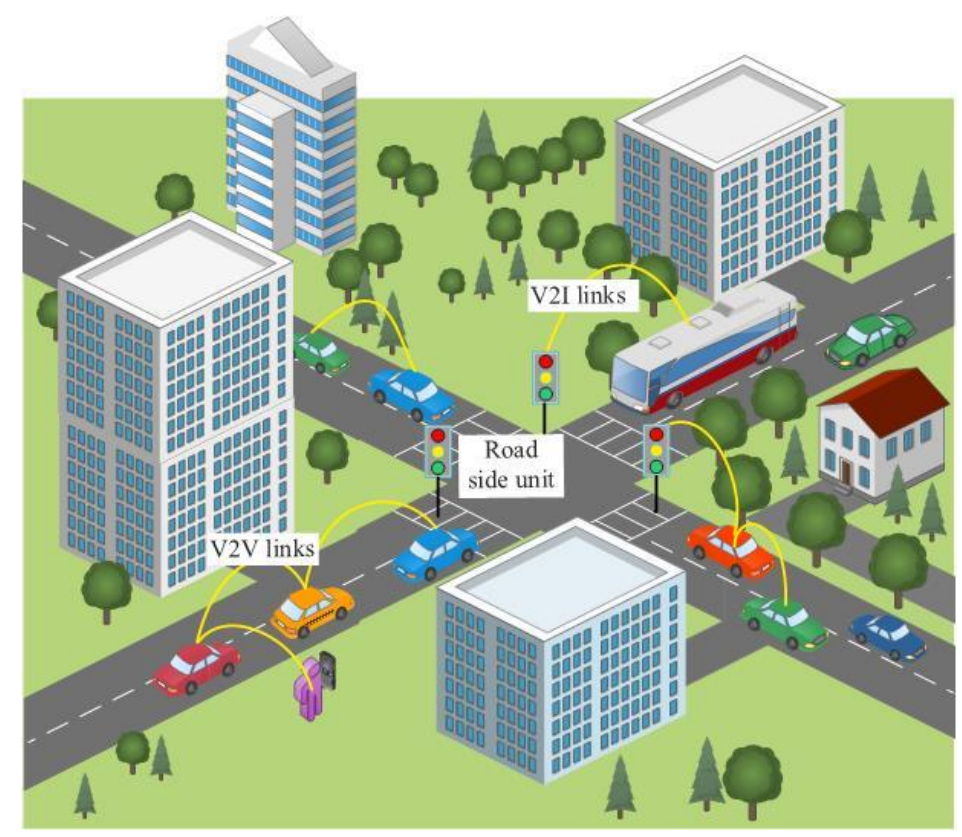

Figure 1. The system model of vehicular network

In this scenario, 5G mmWave and multiple-input-multiple-output (MIMO) provide the position and orientation, respectively. Additionally, accurate location and synchronization information are contributed for the RSUs via vehicle-to-infrastructure (V2I). Vehicle-to-vehicle (V2V) communication is employed for distance measurement and message transformation. The distances model measured with time-difference-of-arrival (TDOA) is given

$$
z_{i j, t}=h_{\mathrm{TDOA}}\left(x_{i, t}, x_{j, t}\right)+\beta_{i, t}+\beta_{j, t}
$$

where $x_{i, t}$ and $\beta_{i, t}$ denote the $i$ vehicle's location and the normally distributed process noise with $N\left(0, \sum_{\beta_{i, t}}\right)$ at the time $t$, respectively. The $h_{\mathrm{TDOA}}\left(x_{i, t}, x_{j, t}\right)$ is a function of both $x_{i, t}$ and $x_{j, t}$.

The mobile state of the vehicle is a random walk model, and each vehicle has a fixed speed and direction. The following state $x_{i, t+1}$ has evolved from the current state $x_{i, t}$ according to

$$
x_{i, t+1}=x_{i, t}+\Delta t \cdot v_{i}+w_{i}
$$

where $\Delta t$ is the time step, $v_{i}$ is the velocity, and $w_{i}$ represents the process noise.

\subsection{Belief Propagation Algorithm for Cooperative Localization}

The cooperative localization algorithm based on the belief propagation (BP) algorithm is widely recognized with high accuracy, and suitable for static and dynamic network scenarios.

To be specific, the BP algorithm is based on factor groups and Gaussian message propagation, which estimates the locations via exchanging the vehicle's statistical information with Gaussian distribution [12,14]. In the beginning, the vehicle broadcasts discovery information and measure distances among neighboring vehicles and RSUs. According to the $i$ vehicle's prior information, the message $\mu$ from factor $f_{i, t}$ to state $x_{i, t}$ at the $l^{\text {th }}$ iteration in time $t$ is given in

$$
\mu_{f_{i, t} \rightarrow x_{i, t}}^{(l)}\left(x_{i, t}\right)=\int p\left(x_{i, t} \mid x_{i, t-1}\right) b^{(l-1)}\left(x_{i, t-1}\right) d x_{i, t-1} .
$$

The vehicle calculates the incoming message when receiving the messages from neighbors, and is given 


$$
\mu_{f_{i j, t} \rightarrow x_{i, t}}^{(l)}\left(x_{i, t}\right)=\int p\left(z_{i j, t} \mid x_{i, t}, x_{j, t}\right) \prod_{j \in S_{i}} \mu_{x_{i, t}^{(l-1)} \rightarrow f_{i j, t}}\left(x_{i, t}\right) d x_{i, t} .
$$

The outgoing message is defined

$$
\mu_{x_{i, t} \rightarrow f_{i j, t}}^{(l)}\left(x_{i, t}\right)=\mu_{f_{i, t} \rightarrow x_{i, t}} \prod_{j \in \mathcal{S}_{i} / j} \mu_{f_{i j, t} \rightarrow x_{i, t}}^{(l)}\left(x_{i, t}\right)
$$

Besides, the posterior distribution $p(x \mid z)$ is denoted

$$
b^{(l)}\left(x_{i, t}\right) \propto \mu_{f_{i, t} \rightarrow x_{i, t}}\left(x_{i, t}\right) \prod_{j \in s_{i}} \mu_{f_{i j} \rightarrow x_{i, t}}^{(l)}\left(x_{i, t}\right)
$$

The location $x_{i, t}$ is estimated by the minimum mean-square error (MMSE) algorithm or maximum a posteriori (MAP) according to $b^{(N i t e r)}\left(x_{i, t}\right)$. The detailed process of the BP algorithm of cooperative localization is presented in Algorithm 1.

Algorithm 1: Belief propagation of cooperative localization

\section{Initialization:}

nodes $i=1: N$ in parallel

The vehicle $i$ discovers its neighboring vehicles and RSUs.

The vehicle $i$ measures the distance between it and its neighbors.

\section{For $l=1$ : Niter}

The vehicle $i$ broadcasts the belief $b^{(l-1)}\left(x_{i, t}\right)$.

The vehicle $i$ receives $b^{(l-1)}\left(x_{j, t}\right)$ from the neighbors.

Vehicle $i$ calculates the incoming and outgoing message.

Vehicle $i$ calculates the new belief $b^{(l)}\left(x_{j, t}\right)$.

end for

end parallel

However, with the number of vehicles increasing, the BP algorithm has high computational complexity and heavy communication overhead. To the vehicle networks, it may cause network congestion, and even be unable to locate.

\subsection{Evolutionary Coalitional Game Algorithm Based Cooperative Localizaion}

Since the Evolutionary coalitional (EC) game is suitable for capturing wireless networks' behaviors in randomness and uncertainties [21-22], we employ it to construct a stable coalition structure.

The EC game for the cooperative localization in vehicle networks is described as

- Players: The finite vehicles join in the game of cooperative localization. The RSUs assist vehicles ' localization but do not play a part in games.

- Population: All vehicles form coalitions, and each coalition belongs to a population.

- Strategy: The vehicle's strategy set is defined in the neighboring coalitions, and the vehicle determines its activities based on the actions of other vehicles.

- Payoff: The vehicle's payoff is influenced by its connected neighbor vehicles. Each vehicle determines which coalition to join according to the preference profile set during the formation and strategy learning stage.

The vehicle's payoff function $u(\bullet)$ is based on [23] and [18], which is composed of the benefit and cost of the vehicle. The $u(\bullet)$ is defined as

$$
u_{i, m}(t)=S P E B_{i, m}+\alpha \cdot \log \left(1-\left(\frac{\left|n_{i}^{(l)}\right|-\Delta}{\mathcal{N}_{i}}\right)^{2}\right)
$$


where $\alpha$ represents the balance parameter; $\mathcal{N}_{i}$ is the number of the $i$ vehicle's neighbors; $n_{i}$ denotes the number of nodes with the $i$ vehicle communication, and $\Delta$ is introduced to avoid $\left|n_{i}^{(l)}\right|=\mathcal{N}_{i}$. The benefit of utility is the squared position error bound (SPEB), which is relevant to the information from anchors, the range information, and the prior knowledge of the vehicles' positions [23-25]. Besides, the cost of the vehicle is the ratio with the number of communication neighbors.

In the vehicle's utility, the number of neighboring vehicles needs to be considered. It is worth noting that more neighbor vehicles can improve the benefit of the node, but the cost also increased. We denote $\boldsymbol{q}_{i}(t)=\left[q_{i, m}\right]_{m \in S_{i}}$ the preference profile set of the $i$ vehicle to select the coalition, which $S_{i}$ is the strategy set of the $i$ vehicle. The evolutionary game dynamics play a significant role in coalition games, which updated the preference profile set for players. It is noticed that the replicator dynamics is a simple and effective dynamic model, and it represents the probability that the player chooses to jump out of the current to another coalition. The definition is followed.

$$
\rho_{i(m, n)}(t)=\frac{1}{n_{i}}\left[\bar{u}_{i, m}(t)-\bar{u}_{i, n}(t)\right]_{+}
$$

where + indicates that a value greater than 0 is selected. In addition to that, the average payoff of the $i$ vehicle is provided via using the payoff function

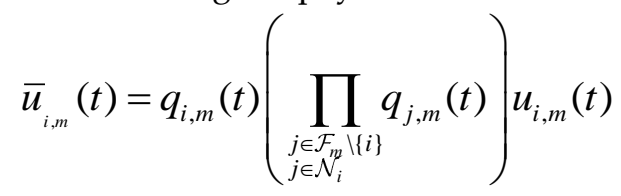

where $q_{i, m}$ represents the probability of the $i$ vehicle selected the $\mathcal{F}_{m}(t)$ coalition, $\mathcal{N}_{i}$ represents the neighboring vehicle set of the $i$ vehicle.

Additionally, the evolution of coalition preference is related to the probability of change and given by

$$
\begin{gathered}
\dot{q}_{i, m}(t)=\sum_{\mathcal{F}_{n} \in \mathcal{S}_{i} \backslash \mathcal{F}_{m}} \rho_{i(n, m)}\left(x_{-i}\right) q_{i, n}(t)-q_{i, m}(t) \sum_{\mathcal{F}_{n} \in \mathcal{S}_{i} \backslash \mathcal{F}_{m}} \rho_{i,(m, n)}\left(x_{-i}\right) \\
q_{i, m}(t+1)=q_{i, m}(t)+\dot{q}_{i, m}(t)
\end{gathered}
$$

If the evolution of coalition preference $\dot{q}_{i, m}$ is greater than 0 , the $i$ vehicle joins in the $m$ coalition. If $\dot{q}_{i, m}$ is equal to 0 , the $i$ vehicle keeps the current coalition. Otherwise, the $i$ vehicle departs the $m$ coalition.

The new strategic preferences based on continuous iterations are provided via the learning stage and a stable coalition structure. The evolutionary stable coalitional structure (ESCS) [26] is employed to represent the stable state of the EC game, in which the vehicle coalitions are resilient to vehicle topology changes. Additionally, the perturbation of strategies is the vehicles' changing due to the joining of new vehicles, NLOS in a vehicle-vehicle link, the message congestion of vehicles, or vehicle exiting location. The ESCS is defined

$$
\bar{u}_{i}\left(\boldsymbol{q}_{i}^{\prime},(1-\epsilon) \boldsymbol{q}_{-i}^{*}+\epsilon \boldsymbol{q}_{-i}^{\prime}\right) \leq \bar{u}_{i}\left(\boldsymbol{q}_{i}^{*},(1-\epsilon) \boldsymbol{q}_{-i}^{*}+\epsilon \boldsymbol{q}_{-i}^{\prime}\right),
$$

where $\epsilon \in(0,1)$ is a probability, $\boldsymbol{q}_{i}^{\prime}$ and $\boldsymbol{q}_{i}^{*}$ are different strategy sets. Specially, when $\epsilon$ of vehicles in the coalition $\boldsymbol{q}_{-i}^{*}$ change their strategy and quit the coalition $\boldsymbol{q}_{-i}^{*}$ or $\epsilon$ portion of vehicles join in this coalition, the vehicles in the coalition $\boldsymbol{q}_{i}^{*}$ still maintain the highest payoff.

The detailed illustration of cooperative localization with the EC game is revealed in Algorithm 2. We employ the EC game to select the neighboring vehicles to reduce the computational complexity and communication overhead. Vehicles form coalitions and exchange information with the member of the coalition. While the number of iterations 
for cooperative localization is maintained, we have reduced the number of communication vehicles.

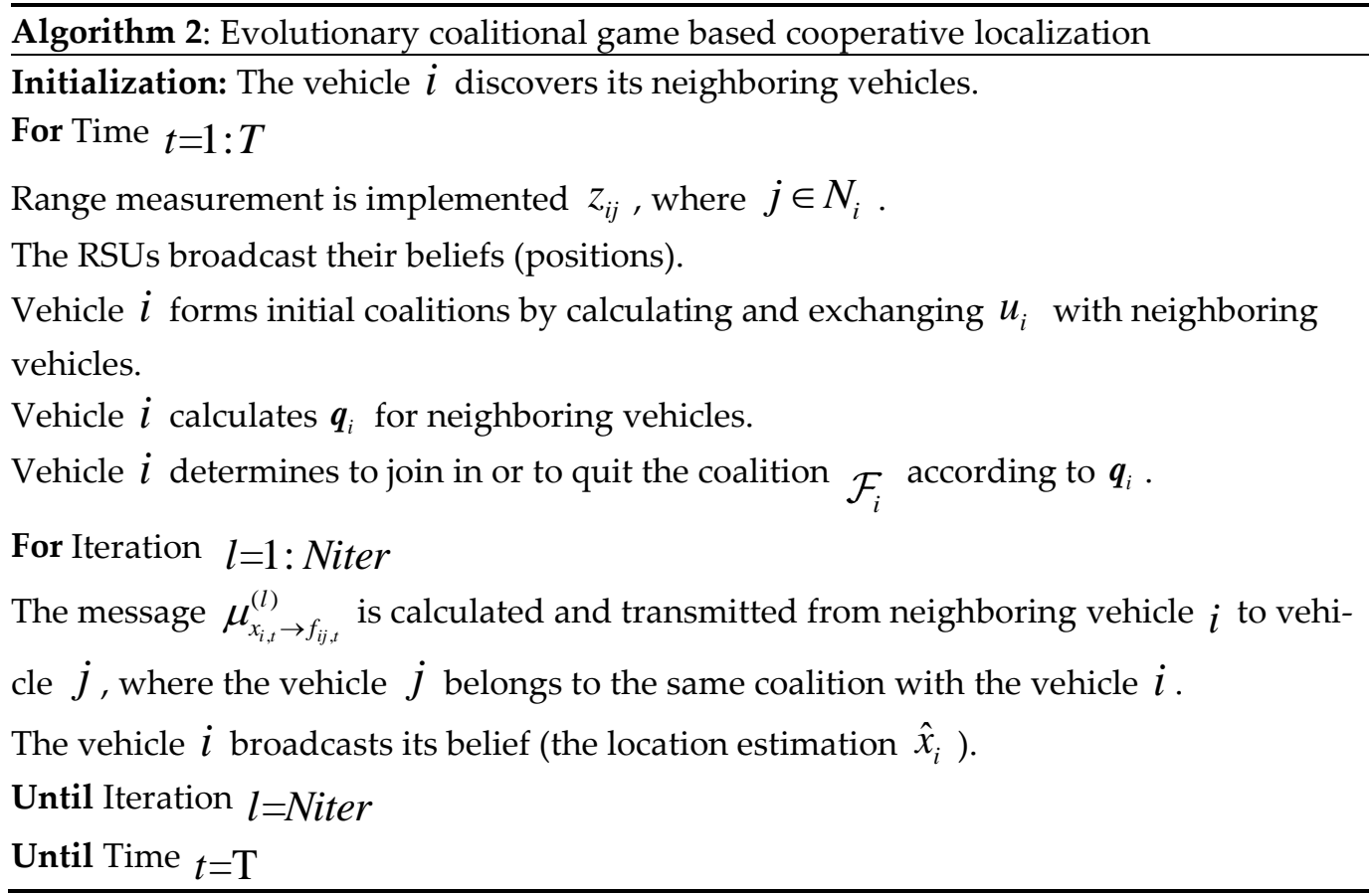

\section{Results}

The simulation deploys a $240 \mathrm{~m} \times 240 \mathrm{~m}$ crossroad with 20 fixed RSUs along the road and 80 randomly placed vehicles, where the distance between two RSUs is $40 \mathrm{~m}$. The considered scenario is described in Fig.2. The red triangle regions are the RSUs, and the blue circles represent the vehicles' positions. The communication range is 30 meters. The vehicles carry out the same direction of movement with different speeds (rang in 0$1 \mathrm{~m} / \mathrm{s}$ ) under the considered scenario. In addition to that, the distance between vehicles is set greater than $6 \mathrm{~m}$ because the size of the vehicle is usually $5 \mathrm{~m}$ long and $2 \mathrm{~m}$ wide. We assume that the coverage of RSUs is seamless, and each vehicle finds one RSU at least. The $\alpha$ is set to 1 , which the two terms of payoff function can be well balanced.

Additionally, the RSU's position is drawn from a Gaussian posterior probability density function (PDF) whose mean is the true position and covariance is $0.1 \mathrm{~m}$. For the vehicle, its covariance is $10 \mathrm{~m}$. The measurement error $\mathrm{R}$ is $1 \mathrm{~m}$. The PLBP with a weight $1 / 3$ and dimension $4 \times 9$ sigma-points is employed to estimate the vehicles' positions. In the end, the $\mathrm{N}_{\text {iter }}$ is set to 5 . 


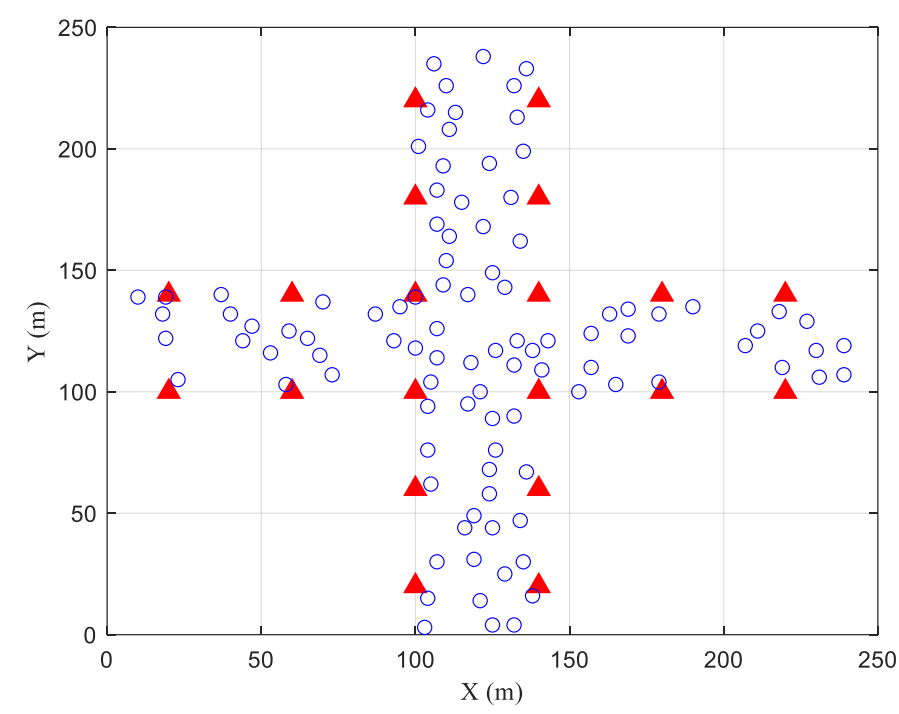

Fig. 2 Scenario for the simulations.

Summarily, each vehicle has a priori location with low precision that obeys the Gaussian distribution. The vehicles learn coalition strategy and form relatively stable coalitions for location estimation utilizing the proposed method. The results are compared with PLBP, which performs well for mobile networks and nonlinear measurements.

1) Localization performance

The RMSE of the proposed method over time in vehicle networks is shown in Fig. 3. Since the EC game achieve the ESCS, the positioning error of the proposed algorithm is larger than that of PLBP in the first few seconds. Fig. 3 demonstrates that the RMSE in the proposed method and PLBP algorithm tends to coverage under time increased. It is noticed that the localization performance degradation is minor. According to the analysis method in Ref [18], the difference from the PLBP method can be neglected.

Meanwhile, the vehicles with different speed are described in Fig.3. Although the speed of the vehicle has an effect on the localization error, whose reason is that the localization algorithm does not utilize the velocity information of past moments, the proposed method has the same trend with the PLBP. Therefore, the proposed method does not affect the localization accuracy at the same speed.

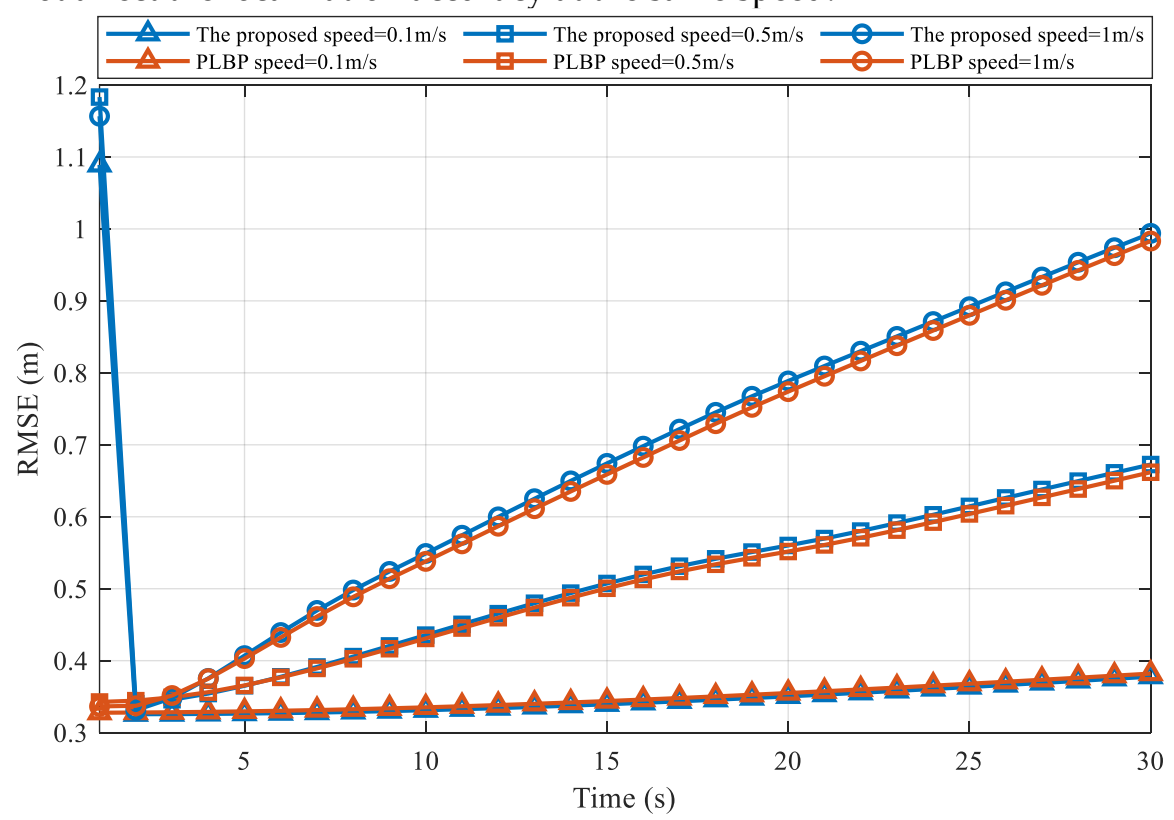

Figure 3. RMSE with different vehicles' speed for cooperative localization

2) Complexity and Network Traffic 
The complexity of cooperative localization algorithm is $\mathcal{O}\left(Q\left|\mathbf{n}_{i}^{(i)}\right|\right)$, where $n_{i}$ denotes the average number of nodes positioned for the $i$ vehicles, and $\mathrm{Q}$ denotes the message representation complexity pre link. The complexity depends on the number of nodes communicated per vehicle for a specific vehicle. Fig.4 describes the comparison of $n_{i}$ between the proposed method and PLBP with iteration of 5. The average number of nodes with proposed EC game-based cooperative localization method is 2, which is less than the PLBP algorithm. The complexity of the proposed method is less than the conventional algorithm.

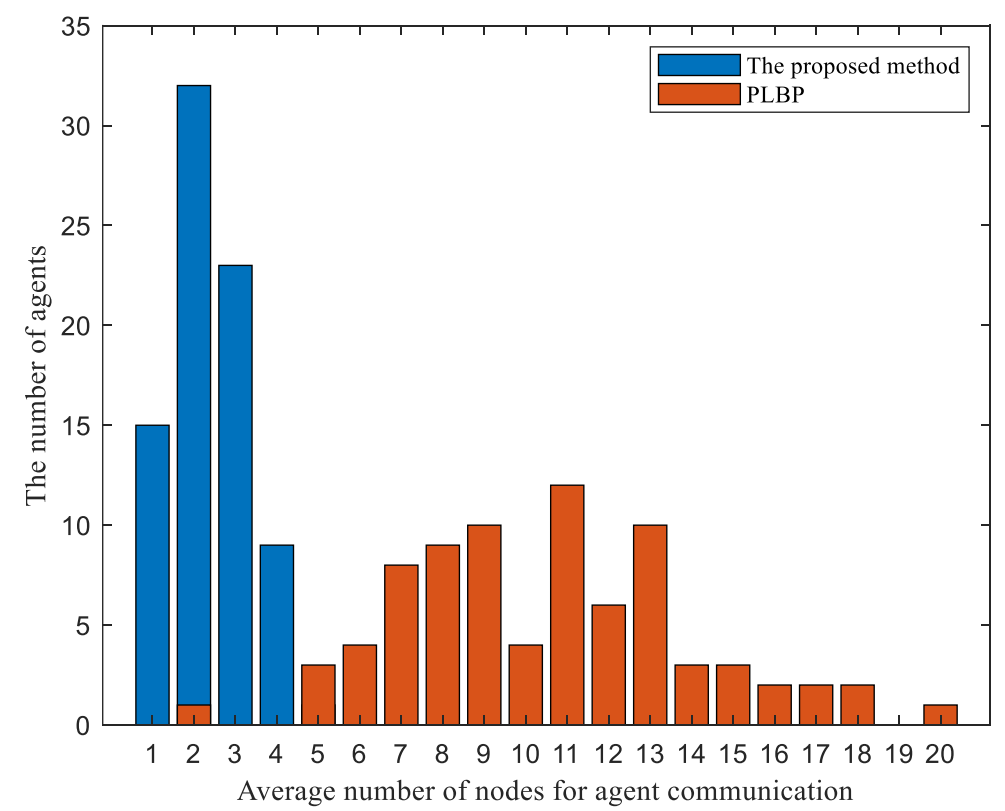

Figure 4. Preference of potential coalition sizes for all vehicles

\section{Discussion}

Inaccurate positions, time synchronization, and erroneous measurement are the primary error sources in wireless localization [27]. Here, we discuss and analyze the influence of different error sources on localization performance. In the proposed localization method, we consider the SPEB and communication cost as the vehicle's payoff of the EC game to form a localization coalition. The SPEB is composed of the RSU's location, range measurement information, and a priori information of the vehicle. Besides, communication cost is relevant to the number of neighbors in the coalition. Since the RSUs as infrastructure has precise location information, we consider the proposed localization method's error sources: time synchronization and measurement error. Therefore, the impaction of above error sources is discussed.

For time synchronization, generally, users within the coverage area of a BS are time-synchronized with the BS in a cellular network. In V2V communication, vehicles within the coverage of the RSU are also synchronized with the RSU, and the vehicles in semi-coverage or outside the coverage are synchronized with the vehicles that need to communicate [28]. However, since the requirements for time synchronization in positioning are much higher than those in communication, an effective time synchronization method is necessary. Similar to the BP algorithm, this proposed method is also affected by time synchronization [32]. The joint synchronization and localization algorithm can exchange the clock parameters of the vehicles while the positioning information is exchanged between the vehicles, thereby ensuring the time synchronization between the vehicles [29-31]. The above method can well solve the impact of time synchronization on cooperative localization, we will not discuss it in detail here. 
Moreover, the measurement error mainly affects the vehicle's SPEB. The SPEB of the vehicle is defined to be

$$
\mathrm{SPEB}_{k} \triangleq \operatorname{tr}\left\{\left[\mathbf{J}_{e}\left(\mathbf{P}_{k}\right)\right]_{2 \times 2, k}^{-1}\right\}
$$

And the $\mathbf{J}_{e}\left(\mathbf{P}_{k}\right)$ is given by

$$
\mathbf{J}_{\mathrm{e}}\left(\mathbf{p}_{k}\right)=\sum_{j \in N_{a, b}} \lambda_{k j}\left(\left(1-\gamma^{2}\right) \beta^{2} \sum_{m=1}^{N_{t}} \mathbf{J}_{\mathbf{y}}\left(\phi_{k j}\right)+\frac{N_{t}\left(\gamma \beta+f_{c}\right)^{2} G_{k j}}{z_{k j}^{2}} \mathbf{J}_{\mathbf{y}}\left(\phi_{k j}+\frac{\pi}{2}\right)\right) \text {. }
$$

From the formula, the distance $z_{k j}$ and the angle $\phi_{k j}$ are variables that need to be measured. Therefore, it is affected by measurement errors.

The measurement error is set to $0 \mathrm{~m}, 1 \mathrm{~m}$, and $10 \mathrm{~m}$ with $0.1 \mathrm{~m} / \mathrm{s}$ speed of vehicles. Fig. 5 shows the RMSE of the proposed method with the measurement error. We observe the influence of the measurement error on the localization accuracy of the vehicles. Note that the proposed localization method is little affected by measurement errors, which is mainly due to the special network topology and the effective cooperative localization algorithm. The roadside unit enables the vehicle to receive precise position information within a range of up to two hops, which greatly reduces error accumulation.

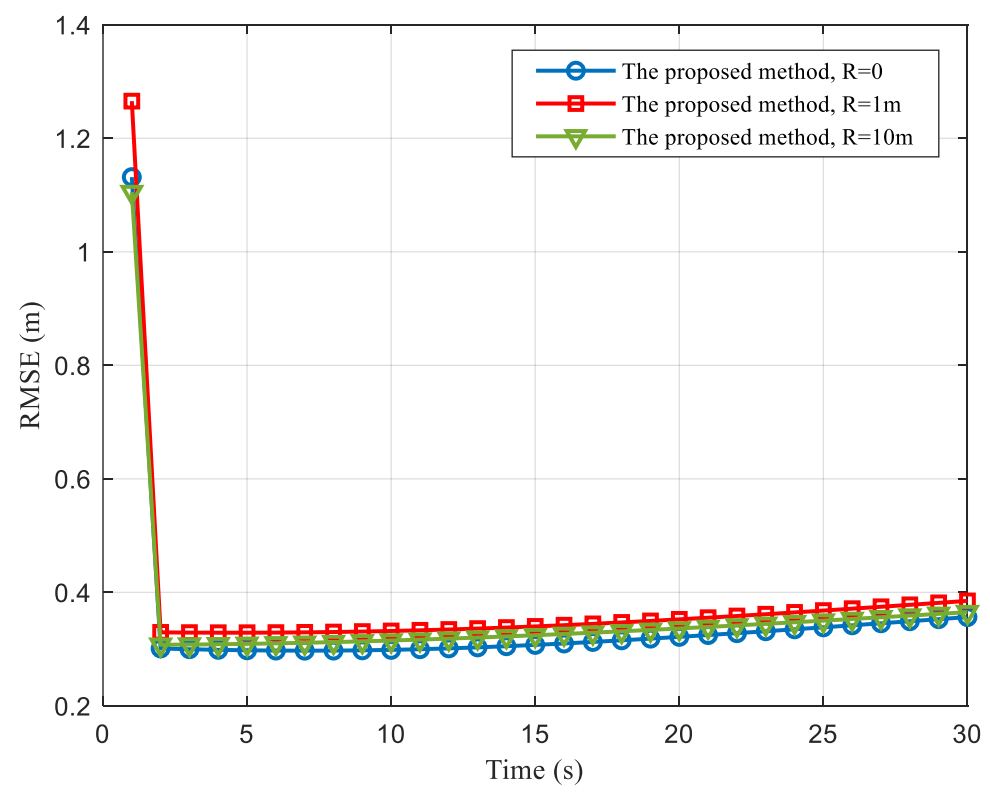

Figure 5. The RMSE with the measurement error

\section{Conclusions}

In this paper, we focused on the limitation of cooperative localization in vehicle networks and proposed a fusion framework based on the cooperative localization and evolutionary coalitional game. Specifically, to reduce the high computational complexity and communication overhead, we carried out the evolutionary coalitional game to select the neighboring vehicles to form coalitions. The vehicle implements positioning via information exchange among neighboring nodes in the coalition. The simulation results indicate that the proposed evolutionary coalitional game based cooperative localization model performed better than the PLBP model. The proposed method reduced the computational complexity and communication overhead while maintaining localization accuracy. The proposed model results are beneficial for vehicles position.

Supplementary Materials: The following supporting information can be downloaded at: www.mdpi.com/xxx/s1, Figure S1: title; Table S1: title; Video S1: title.

Author Contributions: Conceptualization, T. Y. and D. Z.; methodology, T. Y.; software, T. Y.; validation, T. Y.; formal analysis, T. Y.; investigation, T. Y.; resources, T. Y.; data curation, T. Y.; writ- 
ing-original draft preparation, T. Y.; writing-review and editing, C. B.; visualization, C. B.; supervision, X. L.; project administration, X. L.; funding acquisition, D. Z. All authors have read and agreed to the published version of the manuscript.

Funding: This research is supported by National Natural Science Foundation of China under Grant No: 41974032.

Data Availability Statement: The data used to support the findings of this study are available from

the corresponding author upon request.

Acknowledgments: In this section, you can acknowledge any support given which is not covered by the author contribution or funding sections. This may include administrative and technical support, or donations in kind (e.g., materials used for experiments).

Conflicts of Interest: The authors declare no conflict of interest.

\section{References}

1. F. Bounini, D. Gingras, H. Pollart, and et. al. From Simultaneous Localization and Mapping to Collaborative Localization for Intelligent Vehicles. IEEE Intell. Transp. Syst. Mag. 2021, 13, 196-216.

2. Z. Cao, D. Yang, K. Jiang and S. Xu. Monocular Connected-Vehicle Position Estimation on Sloping and Uneven Roads. IEEE Intell. Transp. Syst. Mag. 2022, 14, 228-241.

3. D. Liu, Y. Cui, X. Guo, and et. al. Visual Localization for Autonomous Driving: Mapping the Accurate Location in the City Maze. In Proceedings of the 2020 25th International Conference on Pattern Recognition (ICPR), Milan, Italy, 10-15 Jan. 2021, pp. 3170-3177.

4. W. Yuan, F. Liu, C. Masouros, and et. al. Bayesian Predictive Beamforming for Vehicular Networks: A Low-Overhead Joint Radar-Communication Approach. IEEE Trans. Wireless Commun. 2021, 20, 1442-1456.

5. Z. Z. M. Kassas, M. Maaref, J. J. Morales, and et. al. Robust Vehicular Localization and Map Matching in Urban Environments Through IMU, GNSS, and Cellular Signals. IEEE Intell. Transp. Sys. Mag. 2020, 12, 36-52.

6. A. Eskandarian, C. Wu and C. Sun. Research Advances and Challenges of Autonomous and Connected Ground Vehicles. IEEE Trans. Intell. Transp. Sys. 2021, 22, 683-711.

7. H. Wymeersch, G. Seco-Granados, and et. al. 5G mmWave Positioning for Vehicular Networks. IEEE Wireless Communications 2017, 24, 80-86.

8. 3GPP. NG Radio Access Network (NG-RAN); User Equipment (UE) positioning in NG-RAN. 3GPP TS38.305.

9. H. Kim, K. Granstrom, L. Gao, G. Battistelli, and et. al. 5G mmWave Cooperative Positioning and Mapping Using MultiModel PHD Filter and Map Fusion. IEEE Trans. Wireless Commun. 2020, 19, 3782-3795.

10. F. Luo, S. Wang, Y. Gong, and et. al. Geographical Information Enhanced Cooperative Localization in Vehicular Ad-Hoc Networks. IEEE Signal Process. Lett. 2018, 25, 556-560.

11. W. Meng, X. Chu, Z. Lu, and et. al. V2V Communication Assisted Cooperative Localization for Connected Vehicles. In Proceedings of the 2021 IEEE Wireless Communications and Networking Conference (WCNC), Nanjing, China, 29 March-1 April 2021.

12. H. Wymeersch, J. Lien, and M. Z. Win. Cooperative Localization in Wireless Networks. Proc. IEEE 2009, 97, 427-450.

13. A. T. Ihler, J. W. Fisher, R. L. Moses, and et. al. Nonparametric belief propagation for self-localization of sensor networks. IEEE J. Sel. Areas Commun. 2005, 23, 809-819.

14. Á. F. García-Fernández, L. Svensson and S. Särkkä. Cooperative Localization Using Posterior Linearization Belief Propagation. IEEE Trans. Veh. Technol. 2018, 67, 832-836.

15. Tang, C.; Dou, L. An Improved Game Theory-Based Cooperative Localization Algorithm for Eliminating the Conflicting Information of Multi-Sensors. Sensors 2020, 20, 5579.

16. Z. Zhao, R. Zhang, X. Cheng, and et. al. Network formation games for the link selection of cooperative localization in wireless networks. In Proceedings of the 2014 IEEE International Conference on Communications (ICC), Sydney, NSW, Australia, 1014 June 2014; pp. 4577-4582.

17. K. Hedhly, M. Laaraiedh, F. AbdelKefi, and et. al. Optimizing communication costs based on novel cooperative localization techniques using coalitional game theory in wireless networks. In Proceedings of the $201814^{\text {th }}$ International Wireless Communications \& Mobile Computing Conference (IWCMC), Limassol, Cyprus, 25-29 June 2018; pp:1043-1046.

18. R. Zhang, Z. Zhao, X. Cheng, and L. Yang. Overlapping Coalition Formation Game Based Opportunistic Cooperative Localization Scheme for Wireless Networks. IEEE Trans. Commun. 2017, 65, 3629 - 3642.

19. Ke. M. Tian, S. Tong, K. Zhang, H. An EKF based overlapping coalition formation game for cooperative wireless network navigation. IET Commun. 2021,15,2407- 2424.

20. Z. Abu-Shaban, H. Wymeersch, T. Abhayapala, and et. al. Distributed Two-Way Localization Bounds for 5G mmWave Systems. In Proceedings of the 2018 IEEE Globecom Workshops (GC Wkshps), Abu Dhabi, United Arab Emirates, 9-13 Dec. 2018; pp. 1-6. 
21. M. A. Khan, H. Tembine, and A. V. Vasilakos. Evolutionary coalitional games: design and challenges in wireless networks. IEEE Wireless Commun. 2012, 19, 50-56.

22. A. Asheralieva, T. Q. S. Quek, and D. Niyato. An Asymmetric Evolutionary Bayesian Coalition Formation Game for Distributed Resource Sharing in a Multi-Cell Device-to-Device Enabled Cellular Network. IEEE Trans. Wireless Commun. 2018, 17, 3752-3767.

23. Y. Shen, H. Wymeersch, and M. Z. Win. Fundamental Limits of Wideband Localization - Part II: Cooperative Networks. IEEE Trans. Inform. Theory 2010, 56, 4981-5000.

24. S. Mazuelas, Y. Shen, and M. Z. Win. Spatiotemporal information coupling in network navigation. IEEE Trans. Inform. Theory 2018, 64, 7759-7779.

25. Y. Xiong, N. Wu, Y. Shen, and M. Z. Win. Cooperative localization in massive networks. IEEE Trans. on Inform. Theory 2021, $1-23$.

26. X. Luo and H. Tembine. Evolutionary coalitional games for random access control. In Proceedings of the 2013 Proceedings IEEE INFOCOM, Turin, Italy, 14-19 April 2013; pp. 535-539.

27. W. Yuan, N. Wu, Q. Guo, and et. al. TOA-Based Passive Localization Constructed Over Factor Graphs: A Unified Framework. IEEE Trans. Commun. 2019, 67, 6952-6965.

28. 3GPP. Group Radio Access Network; Evolved Universal Terrestrial Radio Access (E-UTRA) and Evolved Universal Terrestrial Radio Access Network (E-UTRAN); Technical Specification Group Radio Access Network. TS 36.331.

29. W. Yuan, N. Wu, B. Etzlinger, and et. al. Cooperative Joint Localization and Clock Synchronization Based on Gaussian Message Passing in Asynchronous Wireless Networks. IEEE Trans. Veh. Technol. 2016, 65, 7258-7273.

30. B. Etzlinger, F. Meyer, F. Hlawatsch, and et. al. Cooperative Simultaneous Localization and Synchronization in Mobile Agent Networks. IEEE Trans. Signal Process. 2017, 65, 3587-3602.

31. W. Yuan, J. Yuan, D. Wing Kwan Ng. Parametric Message-Passing for Joint Localization and Synchronization in Cooperative Networks. In Proceedings of the 2020 IEEE Global Communications Conference (GLOBECOM), Taipei, Taiwan, 7-11 Dec. 2020 pp. 1-6.

32. Y. Xiong, N. Wu, Y. Shen and et. al. Cooperative Network Synchronization: Asymptotic Analysis. IEEE Trans. Signal Process. $2018,66,757-772$. 